\title{
Adsorption of Xe atoms on metal surfaces: New insights from first-principles calculations
}

\author{
Juarez L. F. Da Silva ${ }^{1}$, Catherine Stampf ${ }^{1,2}$, and Matthias Scheffler ${ }^{1}$ \\ ${ }^{1}$ Fritz-Haber-Institut der Max-Planck-Gesellschaft, Faradayweg 4-6, D-14195 Berlin-Dahlem, Germany \\ ${ }^{2}$ Department of Physics and Astronomy, Northwestern University, Evanston, IL 60208, USA
}

(February 1, 2008)

\begin{abstract}
The adsorption of rare gases on metal surfaces serve as the paradigm of weak adsorption where it is typically assumed that the adsorbate occupies maximally coordinated hollow sites. Densityfunctional theory calculations using the full-potential linearized augmented plane wave method for Xe adatoms on $\mathrm{Mg}(0001), \operatorname{Al}(111), \operatorname{Ti}(0001), \mathrm{Cu}(111), \operatorname{Pd}(111)$, and $\operatorname{Pt}(111)$, show, however, that Xe prefers low-coordination on-top sites in all cases. We identify the importance of polarization and a site-dependent Pauli repulsion in actuating the site preference and the principle nature of the rare-gas atom-metal surface interaction. Copyright (2002) by The American Physical Society.
\end{abstract}

PACS numbers:68.35.Bs,68.35.Md,71.15.Ap

The adsorption of rare-gas atoms on metal surfaces represent prototypical physical adsorption systems [1,2]. Noting that these adatoms have closed shells, it is typically assumed that the adatom-substrate interaction is determined by an interplay of van der Waals attraction and Pauli repulsion, and that these adsorbates prefer maximally coordinated sites [1]. However, the concept of the van der Waals interaction (dispersion forces) is valid for large distances where orbitals almost do not overlap, and at closer distances, near the equilibrium geometry of an adsorbate on a surface, the direct interaction of adsorbate and substrate orbitals can be significant. Thus, although the van der Waals tail is not described by state-of-the-art exchange-correlation functionals, the short-range attraction, as well as the Pauli repulsion, is treated properly. This appears to be valid in particular for the adsorption of heavier rare-gas atoms, but also for the scattering of $\mathrm{He}$ and $\mathrm{Ne}$ atoms at metal surfaces, the interatomic distances at the turning point are sufficiently small that density-functional theory (DFT) calculations provide the correct description [3].

With regard to the preferred adsorption site, about ten years ago it was argued that Xe on $\mathrm{Pt}(111)$ adsorbs in low-coordination on-top sites instead of the expected high-coordination hollow sites [4]; a quite surprising, not widely accepted, and certainly not understood suggestion. This assessment was based on Heatom diffraction data from the low-temperature $(T \leq$ $60 \mathrm{~K}$ ) uniaxially compressed phase of Xe on $\mathrm{Pt}(111)[5,6]$. Later, a spin-polarized low-energy electron diffraction (SP-LEED) study of Xe-adsorption "showed" that Xe atoms adsorb preferentially in hollow sites on $\mathrm{Pt}(111)$ [7] and $\operatorname{Pd}(111)$ [8] in the $(\sqrt{3} \times \sqrt{3}) R 30^{\circ}$ structure (from now on labeled $\sqrt{3}$ ). These latter findings were subsequently contradicted by recent LEED studies, which reported that $\mathrm{Xe}$ in the $\sqrt{3}$ structure on $\mathrm{Cu}(111), \operatorname{Pd}(111)$, and $\mathrm{Pt}(111)$ adsorbs in the on-top sites [9].

Thus, despite the conceived "simplicity" of these adsorption systems, even the most basic questions have not been answered satisfactorily: What is the adsorption site and why is it what it is? and what is the nature of the adsorbate-surface bond?

With respect to first-principles studies, the first goodquality calculation was performed by Müller [10], who studied Xe on $\mathrm{Pt}(111)$ using the cluster approach and the local-density approximation (LDA). It was concluded that the on-top site was energetically preferred over the hollow site by $30 \mathrm{meV}$. The interaction was described in terms of a hybridization of Xe $5 p$ orbitals with metal $d$-states, with the on-top site affording the greater mixing and hence being the preferred adsorption site. Some questions about this work were raised (e.g. [9]): (i) Besides the general concern about the validity of the cluster approach, such calculations may only relate to low coverages, and not to the case of Xe in the $\sqrt{3}$ adlayer, which is the only structure for which good experimental data exists. (ii) It is also unclear why the top site affords the greater mixing. As it stands, this is more a statement than an explanation. (iii) Because on-top site adsorption was also found for $\mathrm{Xe}$ on $\mathrm{Cu}(111)$, for which the $d$-band lies well below the Fermi level, a crucial role of substrate $d$-states seems unlikely [9]. A recent work by Betancourt and Bird [11] find that Xe also prefers the on-top site in the $\sqrt{3}$ adlayer structure on $\mathrm{Pt}(111)$. And for an artificial $c(2 \times 2)-\mathrm{Xe} / \mathrm{Ag}(001)$ structure, Clarke et al. [12] reported a theoretical study which also favors the on-top site. However, neither Betancourt and Bird, nor Clarke et al. presented a consistent explanation for the on-top site preference. Finally, a recent study of the $\mathrm{Xe} / \mathrm{Cu}(111)$ system using a ten-atom cluster model, has investigated the interface dipole and work-function, but the site preference was not addressed [13]. 




FIG. 1. DFT-LDA results for: (a) Relative adsorption energy, $\Delta E_{\text {ad }}$, of $\mathrm{Xe}$ on $\operatorname{Pt}(111)$ (the energy of the on-top site is the energy zero). The inset shows a top view of the fcc(111)- $(1 \times 1)$ surface unit cell, indicating the six different sites considered for Xe-adsorption. Full circles denote the sites for the Xe atoms, the large open, pale, and dark gray circles denote the metal atoms in the first, second, and third layers, respectively. (b) Work-function change, $\Delta \Phi$. The calculated value for the clean surface is $\Phi^{\mathrm{Pt}(111)}=6.06 \mathrm{eV}$.

To determine the adsorption site preference of $\mathrm{Xe}$ adatoms on metal surfaces, to explain the site preference, and to provide an improved understanding of rare-gas adsorption on metal surfaces in general, we performed DFT calculations using the all-electron, fullpotential linearized augmented plane wave (FP-LAPW) method [14,15] for Xe adatoms on the $\mathrm{Mg}(0001), \mathrm{Al}(111)$, $\mathrm{Ti}(0001), \mathrm{Cu}(111), \mathrm{Pd}(111)$, and $\mathrm{Pt}(111)$ surfaces. We employed two different exchange-correlation functionals, namely, the LDA [16] and the generalized gradient approximation (GGA) [17]. The surfaces are treated by the supercell approach using the repeated slab geometries (five- and six-layer thick slabs separated by a vacuum region of $18 \AA$ ). To determine the potential-energy surface (PES) of Xe on the metal surfaces we chose six sites on the fcc(111) surface: on-top, bridge, fcc, hcp, the midpoint between on-top and fcc, and the midpoint between on-top and hcp (see inset of Fig. 1). For each site the height of the $\mathrm{Xe}$ atom and the position of the topmost two substrate layers are fully relaxed.

Our calculations show: $(i)$ The on-top site is energetically favored for all studied systems in the $\sqrt{3}$ structure. (ii) Calculations for low Xe coverage, $\Theta_{\mathrm{Xe}}=1 / 4$ and $1 / 9$, show that also in these cases Xe atoms prefer the on-top site (e.g., for $\mathrm{Xe} / \mathrm{Pd}(111)$ and $\mathrm{Xe} / \mathrm{Pt}(111)$ the on-top site is favored over the fcc site by $20.59 \mathrm{meV}$ and $57.87 \mathrm{meV}$, respectively, at $\Theta_{\mathrm{Xe}}=1 / 9$ using the LDA). Thus, lateral $\mathrm{Xe}-\mathrm{Xe}$ interactions are not responsible for the on-top Xe
TABLE I. Adsorption energy of Xe in the on-top site, $E_{\text {ad }}$; adsorption-energy difference between on-top and fcc sites, $E_{\mathrm{ad}}^{\mathrm{ft}}$ $=E_{\mathrm{ad}}^{\mathrm{fcc}}-E_{\mathrm{ad}}^{\mathrm{on}-\mathrm{top}}$ relative to the on-top site; work-function change, $\Delta \Phi$; vertical distance of Xe to the first metal layer, $d_{\mathrm{Xe}-\mathrm{metal}}$ (given as the average distance for the topmost layer), and substrate rumpling (a positive sign indicates an outward and a negative sign an inward displacement). In parenthesis are experimental results $[1,9]$.

\begin{tabular}{|c|c|c|c|c|}
\hline & \multirow{2}{*}{$\begin{array}{c}E_{\mathrm{ad}} \\
(\mathrm{meV})\end{array}$} & \multirow{2}{*}{$\begin{array}{l}\Delta E_{\mathrm{ad}}^{\mathrm{ft}} \\
(\mathrm{meV})\end{array}$} & \multicolumn{2}{|c|}{$\Delta \Phi(\mathrm{eV})$} \\
\hline & & & on-top & fcc \\
\hline$\overline{\operatorname{Mg}(0001)}$ & -130 & 4.34 & -0.22 & -0.16 \\
\hline $\mathrm{Al}(111)$ & -176 & 1.12 & -0.38 & -0.33 \\
\hline $\operatorname{Ti}(0001)$ & -196 & 21.82 & -0.87 & -0.70 \\
\hline $\mathrm{Cu}(111)$ & $-277(-190)$ & 9.14 & -0.96 & -0.83 \\
\hline $\operatorname{Pt}(111)$ & $-367(-320)$ & 49.09 & -1.36 & -0.98 \\
\hline \multirow{3}{*}{$\operatorname{Pd}(111)$} & $-453(-360)$ & 51.22 & -1.44 & -1.28 \\
\hline & \multicolumn{2}{|c|}{$\overline{d_{\text {Xe-metal }}(\AA)}$} & \multirow{2}{*}{\multicolumn{2}{|c|}{$\begin{array}{c}\text { substrate rumpling }(\ddot{A}) \\
\text { on-top }\end{array}$}} \\
\hline & on-top & fcc & & \\
\hline$\overline{\mathrm{Mg}(0001)}$ & 3.80 & 3.84 & 0.00 & \\
\hline $\mathrm{Al}(111)$ & 3.69 & 3.72 & -0.01 & \\
\hline $\mathrm{Ti}(0001)$ & 3.56 & 3.66 & +0.06 & \\
\hline $\mathrm{Cu}(111)$ & $3.25(3.60)$ & 3.31 & +0.03 & $1 \pm 0.02)$ \\
\hline $\operatorname{Pt}(111)$ & $3.07(3.40)$ & 3.19 & +0.04 & $1 \pm 0.02)$ \\
\hline $\operatorname{Pd}(111)$ & $2.85(3.07)$ & 2.86 & +0.05 & $2 \pm 0.03)$ \\
\hline
\end{tabular}

site preference. (iii) Spin-orbit coupling corrections were included for the valence-states using the second variational method [14]; this yields a change in the energy difference between on-top and fcc sites of $\leq 2 \mathrm{meV}$, and the geometrical parameters are unchanged. $(i v)$ The energy difference between the on-top and fcc sites is smaller in the GGA than in the LDA (e.g., for Xe/Pd(111) the ontop site is favorable by $13.48 \mathrm{meV}$ (GGA) and $51.22 \mathrm{meV}$ (LDA), while for $\mathrm{Xe} / \mathrm{Pt}(111)$ the on-top site is favorable by $4.80 \mathrm{meV}$ (GGA) and $49.09 \mathrm{meV}$ (LDA)). Furthermore, the GGA predicts a weaker adsorption energy than LDA (e.g., $E_{\mathrm{ad}}^{\mathrm{Xe} / \mathrm{Pd}(111)}=-76 \mathrm{meV}$ (GGA) and $-453 \mathrm{meV}(\mathrm{LDA}), E_{\mathrm{ad}}^{\mathrm{Xe} / \mathrm{Pt}(111)}=-82 \mathrm{meV}(\mathrm{GGA})$ and $-367 \mathrm{meV}$ (LDA)). However, both functionals gives the same qualitative picture for the interaction between $\mathrm{Xe}$ atoms and metal surfaces. In view of these four points we limit our discussion in the following to the LDA results, neglecting spin-orbit coupling corrections for the valence states, and discussing only the $\sqrt{3}$ structure. All our results, including convergence tests, will be presented in detail in a forthcoming publication [18].

Figures $1 \mathrm{a}$ and $1 \mathrm{~b}$ show respectively the relative binding energy (with respect to the on-top site geometry) and the adsorption induced work-function change for Xe on $\mathrm{Pt}(111)$. It can be seen that the on-top site is energetically favorable and Xe adsorption induces a decrease in the work-function (which is greater for the on-top site), in agreement with experiment. The adsorption energy, the relative energy difference for Xe in the on-top and fcc sites, as well as the work-function change for all the 


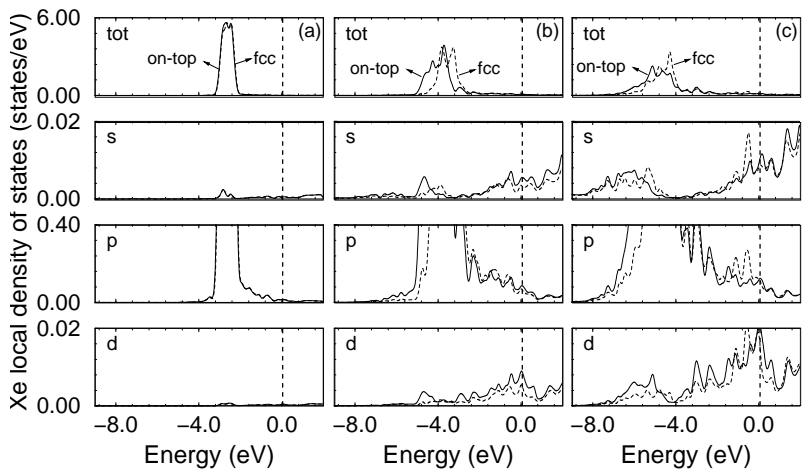

FIG. 2. Local density of states for Xe on $\operatorname{Pt}(111)$ for different vertical positions of Xe, $Z$ : (a) $Z=4.53 \AA$; (b) $Z=$ $3.07 \AA$ (equilibrium Xe position); (c) $Z=2.70 \AA$.

systems are summarized in Tab. I, where it can be seen that this same behavior is exhibited. For the cases where there are experimental values of the adsorption energy with which to compare $(\mathrm{Xe} / \mathrm{Cu}, \mathrm{Xe} / \mathrm{Pd}$, and $\mathrm{Xe} / \mathrm{Pt})$, the agreement between theory and experiment is quite good.

In Tab. I we also list the important structural data. The DFT-LDA bond lengths are about $10 \%$ shorter than those obtained by LEED [9], but in view of the fact that the adsorption energy is weak in physisorption systems and our theory does not include vibrational contributions, the sign and size of the deviation is as expected. The difference in bondlengths, however, for the $\mathrm{Xe} / \mathrm{Cu}, \mathrm{Xe} / \mathrm{Pd}$, and $\mathrm{Xe} / \mathrm{Pt}$ systems, for which comparison is possible, are in excellent agreement between theory and LEED analyses. There is one deviation between the calculations and LEED analyses and that is in the direction of displacement of the atom below the Xe atom. The experimental values lie however within the given error margins. Particularly interesting is that the vertical distance between the Xe adatom and the topmost metal layer is smaller for the on-top site as compared to the fcc site for all the studied cases (for Xe/Pd it is almost equal). This is in contrast to the calculations of Ref. [10], and in contrast to what might be intuitively expected. We will come back to this point below.

Figures 2a, 2b, and 2c show the local density of states (LDOS) for Xe on $\mathrm{Pt}(111)$ for three distances of Xe above the surface. It can be seen that there is a broadening of the Xe states as the Xe adatom approaches the surface, which leads to a partial occupation of the previously unoccupied Xe $6 s$ - and $5 d$-states (mainly $5 d_{\mathrm{z}^{2}}$-state), and a partial depopulation of the Xe $5 p$-states (mainly the $\mathrm{Xe} 5 p_{\mathrm{z}}$-state). The same behavior in the LDOS is identified for Xe on all the studied metal surfaces. We note that in Ref. [19], occupation of the Xe-6s resonance was attributed to being the origin of the Fermi level LDOS which renders Xe visible on $\mathrm{Ni}(110)$ in scanning tunneling microscopy experiments. This is consisent with the

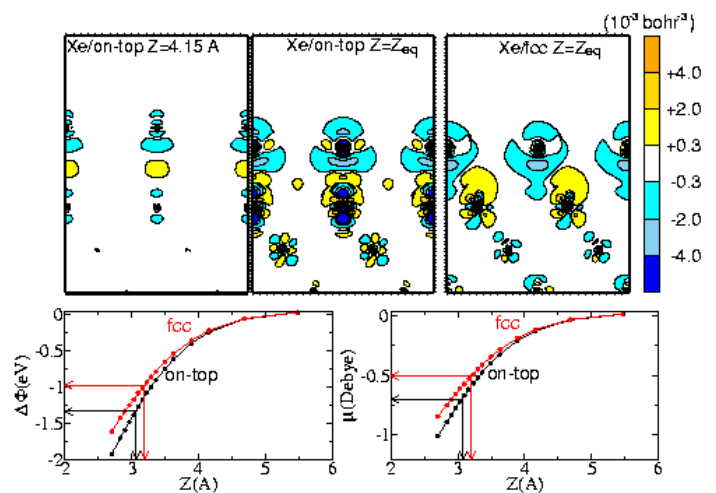

FIG. 3. Upper panels: Difference electron density, $n^{\Delta}(\mathbf{r})=n^{\mathrm{Xe} / \mathrm{Pt}}(\mathbf{r})-n^{\mathrm{Pt}}(\mathbf{r})-n^{\mathrm{Xe}}(\mathbf{r})$, along the $(11 \overline{2})$ plane for $\mathrm{Xe}$ on $\mathrm{Pt}(111)$ in the on-top site at $4.15 \AA$ above the equilibrium position (left), in the on-top equilibrium site $\left(\mathrm{Z}=\mathrm{Z}_{\mathrm{eq}}\right)$ (middle), and in the fcc-hollow equilibrium site (right). Lower panels: Work-function change, $\Delta \Phi$, (left) and dipole moment, $\mu$, (right) versus distance of Xe from the surface. The points are the DFT-LDA values.

present results.

We now turn to consider the change in electron density due to adsorption, i.e., the difference between the density of the interacting system, $n^{\mathrm{Xe} / \mathrm{Pt}}$, and the sum of the densities of the clean surface, $n^{\mathrm{Pt}}$, and a free Xe layer, $n^{\mathrm{Xe}}$. We find that a polarization of the Xe atom sets in when it is already far from the surface, where the negative end of the dipole points towards the surface, i.e., the electron density is enhanced closer to the metal surface (see Fig. 3, upper left). This is due to the general property that the potential in front of a metal is attractive for electrons; specifically, the exchange-correlation potential is more attractive on the metal side of the Xe atom than on the vacuum side. At this large distance where the Xe dipole moment starts to build up, the electrostatic and covalent contributions are small, if present at all. This behavior is consistent with the pioneering works of Lang [20] and Antoniewicz [21]. A corresponding polarization occurs in the surface, which can be described as the image of the Xe dipole. The build-up of these dipoles happens simultaneously (and continuously with decreasing distance from the surface) and are related to the partial population and depopulation of the Xe states (cf. Fig. 2). In Fig. 3, the work-function change and dipole moment is shown as a function of distance, Z. For all $\mathrm{Z}$, the values for the on-top site are greater and the variation with distance is smooth and continuous. From the electron density difference distributions at the equilibrium positions $\left(\mathrm{Z}=\mathrm{Z}_{\mathrm{eq}}\right)$ depletions of charge are seen at the Xe atom (mainly $5 p_{z}$ ) and at the metal atom closest to Xe, i.e., mainly in the $\mathrm{Pt} d_{\mathrm{z}^{2}}$-states, while an increase occurs at the surface, between these regions of depletion. Similar results are found for all the other studied transition metal surfaces. 



FIG. 4. Left panel: Calculated binding energies of Xe on $\operatorname{Pt}(111)$ for the on-top and fcc sites as a function of distance from the surface. The filled and open circles indicate DFT-LDA data. Right panel: decomposition into repulsive and attractive contributions (see text). $Z=0$ is the center of the top layer.

The differences in the LDOS and $\Delta n(\mathbf{r})$ distributions between on-top and hollow sites described above, show that the Pauli repulsion and the attractive interaction is not the same at each site. In order to investigate this aspect in more detail, we calculated the binding energy of $\mathrm{Xe}$ on the metal surfaces as a function of vertical distance. The results for $\mathrm{Xe} / \mathrm{Pt}$ are shown in Fig. 4 (left). At all distances above the equilibrium position, the ontop site has lower energy. From these results, we obtain Xe vibrational frequencies of $E_{\mathrm{vib}}=3.79 \mathrm{meV}$ (on-top) and $4.17 \mathrm{meV}$ (fcc). Reported experimental values are $3.70 \mathrm{meV}$ and $3.80 \mathrm{meV}$, [2] showing excellent agreement with the calculated (on-top) value.

In Fig. 4 (right), we decomposed the binding energy curves into a repulsive and attractive potential using a standard empirical formula: $E(Z)=A \exp (-B Z)-$ $C\left(Z-Z_{0}\right)^{-3}[1]$, where $Z_{0}$ is the position of the image plane. We note that the two right-most DFT-LDA points in Fig. 4 (left) were not included in the fit since DFT results are most reliable near the minimum in the potential well. It can be seen quite clearly that the Pauli repulsion is different for the two sites and is weakest for the on-top site. We also used other expressions for the repulsive and attractive terms, and the conclusion was always the same. We furthermore decomposed the DFT-total energy into kinetic, Coulomb, and exchange-correlation energies. The results suggest that the repulsion due to the kinetic energy is smaller for $\mathrm{Xe}$ in the on-top site. We find the same behavior as exhibited in Fig. 4 for all the rare-gas atom-metal systems investigated.

The reason why, that for $\mathrm{Xe}$ at the on-top site, the Pauli repulsion is weaker, the equilibrium distance closer to the surface, and the dipole moment greater, is explained as follows: On analyzing the reactivity index of the clean Pt surface [22], we find that the electronic char- acter at the on-top region is donor-like and at the hollow region it is acceptor like. Thus, for a negative charge, at not too close distance (or for a dipole with the negative end pointing toward the surface), the on-top region can easily screen this perturbation by transferring electron density from the on-top region (i.e., from $d_{z^{2}}$ and $p_{z}$ orbitals) to the interstitial region. Therefore, at intermediate distances the Xe atom becomes polarized more strongly at the on-top geometry, because the screening charge at the metal can build up more efficiently. This is why the dipole is greater. Similarily, at close distance, when wavefunctions start to overlap and Pauli repulsion sets in, the substrate can reduce this repulsion more efficiently at the on-top site by transferring $d_{z^{2}}$ and $p_{z}$ electrons to $s$-states. Hence Xe can get closer to the surface.

In summary, from DFT calculations we determined that Xe adatoms on the free-electron-like, early and late transition metals, as well as noble metals, $\mathrm{Mg}(0001)$, $\mathrm{Al}(111), \mathrm{Ti}(0001), \mathrm{Cu}(111), \mathrm{Pd}(111)$, and $\mathrm{Pt}(111)$, prefer low coordination on-top sites. The qualitative results are the same for LDA and GGA functionals. We find that the dominant mechanisms at play in these systems are polarization and a site-dependent Pauli repulsion which is weaker for the on-top site, thus stabilizing on-top adsorption. Our recent investigations for the smaller raregas atoms, e.g., Ar and $\mathrm{Kr}$ on $\mathrm{Pd}(111)$ exhibit the same behavior and basic mechanisms, and we anticipate that the present findings may also be generally valid for other rare-gas/metal systems, thus presenting a new picture of rare-gas adsorption, where the on-top site is the rule rather than the exception.

[1] L. W. Bruch, M. W. Cole, and E. Zaremba, in Physical Adsorption: Forces and Phenomena, Oxford Science Publications (1997).

[2] P. Zeppenfeld, in Physics of covered solid surfaces I. Adsorbed layers on surfaces, Landolt-Börnstein (to be published), see reference therein.

[3] M. Petersen et al., Phys. Rev. Lett. 76, 995 (1996).

[4] J. M. Gottlieb, Phys. Rev. B 42, 5377 (1990).

[5] K. Kern et al., Solid State Commun. 62, 391 (1987).

[6] P. Zeppenfeld et al., Phys. Rev. B 38, 3918 (1988).

[7] M. Potthoff et al., Surf. Sci 322, 193 (1995).

[8] G. Hilgers, et al., Surf. Sci. 322, 207 (1995).

[9] Th. Seyller et al., Chem. Phys. Lett. 291, 567 (1998); Phys. Rev. B 60, 11084 (1999).

[10] J. E. Müller, Phys. Rev. Lett. 65, 3021 (1990).

[11] A. E. Betancourt and D. M. Bird, J. Phys. Condens. Mat. 12, 7077 (2000).

[12] S. Clarke, G. Bihlmayer, and S. Blügel, Phys. Rev. B 63, 85416, (2001).

[13] P. S. Bagus et al. Phys. Rev. Lett. 89, 096104 (2002).

[14] P. Blaha, K. Schwarz, and J. Luitz, WIEN97, A FullPotential Linearized Augmented Plane Wave Package for Calculating Crystal Properties, (Karlheinz Schwarz, Techn. Univ. Wien, Vienna 1999). ISBN 3-9501031-0-4. 
[15] M. Petersen et al., Comput. Phys. Commun. 126, 294 (2000); R. Dohmen et al., Comput. Sci. \& Engin. 3, 18 (2001).

[16] J. P. Perdew and Y. Wang, Phys. Rev. B 45, 13244 (1992).

[17] J. P. Perdew, S. Burke, and M. Ernzerhof, Phys. Rev. Lett. 77, 3865 (1996).

[18] J. L. F. Da Silva, C. Stampfl, and M. Scheffler, in preparation.

[19] D. M. Eigler et al. Phys. Rev. Lett. 66, 1189 (1991).

[20] N. D. Lang, Phys. Rev. Lett. 46, 842 (1981).

[21] P. R. Antoniewicz, Phys. Rev. Lett. 32, 1424 (1974).

[22] S. Wilke, M. H. Cohen, and M. Scheffler, Phys. Rev. Lett. 77, 1560 (1996). 